\title{
Observation of heavy spin-orbit excitons propagating in a nonmagnetic background: The case of $(\mathrm{Ba}, \mathrm{Sr})_{2} \mathrm{YIrO}_{6}$
}

\author{
M. Kusch, ${ }^{1}$ V. M. Katukuri, ${ }^{2, *}$ N. A. Bogdanov, ${ }^{2, \dagger}$ B. Büchner, ${ }^{1,2}$ T. Dey, ${ }^{2,3}$ D. V. Efremov, ${ }^{2}$ J. E. Hamann-Borrero, ${ }^{2}$ \\ B. H. Kim, ${ }^{2,4,5}$ M. Krisch, ${ }^{6}$ A. Maljuk, ${ }^{2}$ M. Moretti Sala, ${ }^{6}$ S. Wurmehl, ${ }^{2,7}$ G. Aslan-Cansever, ${ }^{2}$ \\ M. Sturza, ${ }^{2}$ L. Hozoi, ${ }^{2}$ J. van den Brink, ${ }^{2,7}$ and J. Geck ${ }^{1}$ \\ ${ }^{1}$ Institute of Solid State and Materials Physics, TU Dresden, 01069 Dresden, Germany \\ ${ }^{2}$ Leibniz Institute for Solid State and Materials Research IFW Dresden, Helmholtzstrasse 20, D-01069 Dresden, Germany \\ ${ }^{3}$ Experimental Physics VI, EKM, University of Augsburg, 86159 Augsburg, Germany \\ ${ }^{4}$ Computational Condensed Matter Physics Laboratory, RIKEN, Wako, Saitama 351-0198, Japan \\ ${ }^{5}$ Interdisciplinary Theoretical Science (iTHES) Research Group, RIKEN, Wako, Saitama 351-0198, Japan \\ ${ }^{6}$ European Synchrotron Radiation Facility, Boîte Postale 220, F-38043 Grenoble Cedex, France \\ ${ }^{7}$ Department of Physics, TU Dresden, 01069 Dresden, Germany
}

(Received 5 July 2017; published 23 February 2018)

\begin{abstract}
We present a combined experimental and theoretical study of the elementary magnetic excitations in $\mathrm{Ba}_{2} \mathrm{YIrO}_{6}$ and $\mathrm{Sr}_{2} \mathrm{YIrO}_{6}$ - the two most intensively discussed candidates for a new type of magnetic instability caused by exciton condensation. For both materials, high-resolution resonant inelastic X-ray scattering (RIXS) at the Ir $L_{3}$ edge reveals sharp excitations around 370 and $650 \mathrm{meV}$ energy loss, which we identify as triplet and quintet spin-orbit excitons. While the momentum-dependent RIXS spectra reveal that both the triplet and the quintet propagate coherently within the nonmagnetic background of the singlet sites, these modes remain fully gapped. The Ir-Ir exchange interactions in both double perovskites are therefore not strong enough to overcome the magnetic gap and, hence, our results exclude an intrinsic magnetic instability due to a condensation of magnetic excitations for both $\mathrm{Ba}_{2} \mathrm{YIrO}_{6}$ and $\mathrm{Sr}_{2} \mathrm{YIrO}_{6}$.
\end{abstract}

DOI: 10.1103/PhysRevB.97.064421

\section{INTRODUCTION}

When kinetic energy, spin-orbit coupling (SOC), and correlation effects in $4 d$ and $5 d$ transition-metal oxides join forces, new states of matter may emerge. Very striking and famous examples of this are provided by the $5 d$ iridium oxides, where the strong SOC promotes electron correlation effects and also gives rise to strongly anisotropic exchange interactions. The latter feature has attracted particular attention, because it enables one to realize novel magnetic quantum systems [1-3] in an actual material, including the one described by the Kitaev model [4]. The iridates hence provide a unique platform to explore unconventional states of matter like quantum spin liquids [4], spin-orbit-assisted Mott transitions [5], nontrivial topological phases [6,7], unconventional superconductivity [8,9], Weyl semimetals, and axion insulators [10,11].

So far, ongoing research in this field largely focused on iridium oxides containing formally $\mathrm{Ir}^{4+}$ sites with a $t_{2 g}^{5}$ configuration. Far less studied in this context are materials where the formal valence of Ir is 5+. Indeed, the local electron-electron and spin-orbit interactions on an $\mathrm{Ir}^{5+}$ site with a $d^{4}$ or, more precisely, $t_{2 g}^{4}$ configuration stabilize a nonmagnetic ground state with $L=1, S=1$, and total angular

\footnotetext{
*Present address: C3MP, IPHYS, École Polytechnique Fédérale de Lausanne (EPFL), CH-1015 Lausanne, Switzerland.

†Present address: Max Planck Institute for Solid State Research, Heisenbergstrasse 1, 70569 Stuttgart, Germany.
}

momentum $J=L-S=0$, as illustrated schematically in Fig. 1(a). Consequently, such systems have not been expected to provide a fertile ground for interesting magnetic phenomena.

It therefore came as a big surprise when peculiar magnetic instabilities were also predicted for $\operatorname{Ir} d^{4}$ systems: a theoretical study by Khaliullin et al. showed that singlet-triplet (site) exchange can lead in such systems to a magnetic instability, which can be understood in terms of $J=1$ excitations propagating in a background of singlet $(J=0)$ sites [cf. Fig. 1(b)] [3]. This propagation may imply a strong momentum dependence of the $J=1$ mode, with an excitation energy that may even vanish for certain momenta, as illustrated in Fig. 1(c). When this happens, a transition from a nonmagnetic to a magnetic phase takes place, through Bose-Einstein condensation of the $J=1$ excitations [12]. Since the low-lying $J=1$ and $J=2$ multiplets are often referred to as spin-orbit excitons [13], the term "excitonic" magnetism has been coined for the resulting magnetic state [3]. ${ }^{1}$

A number of experiments indeed suggest an unexpected magnetic behavior in the $\mathrm{Ir} d^{4}$ double perovskites $\mathrm{Sr}_{2} \mathrm{YIrO}_{6}$ and $\mathrm{Ba}_{2} \mathrm{YIrO}_{6}$ [15-17]. However, the origin of this magnetism remains a matter of intense debate: while it has been argued that in $\mathrm{Sr}_{2} \mathrm{YIrO}_{6}$ a large crystal-field (CF) splitting and strong SOC do generate excitonic magnetism at low temperatures,

\footnotetext{
${ }^{1}$ Multipolar ordering due to the condensation of $J>1$ excitations is also discussed in other physical systems such as heavy-fermion compounds [14].
} 

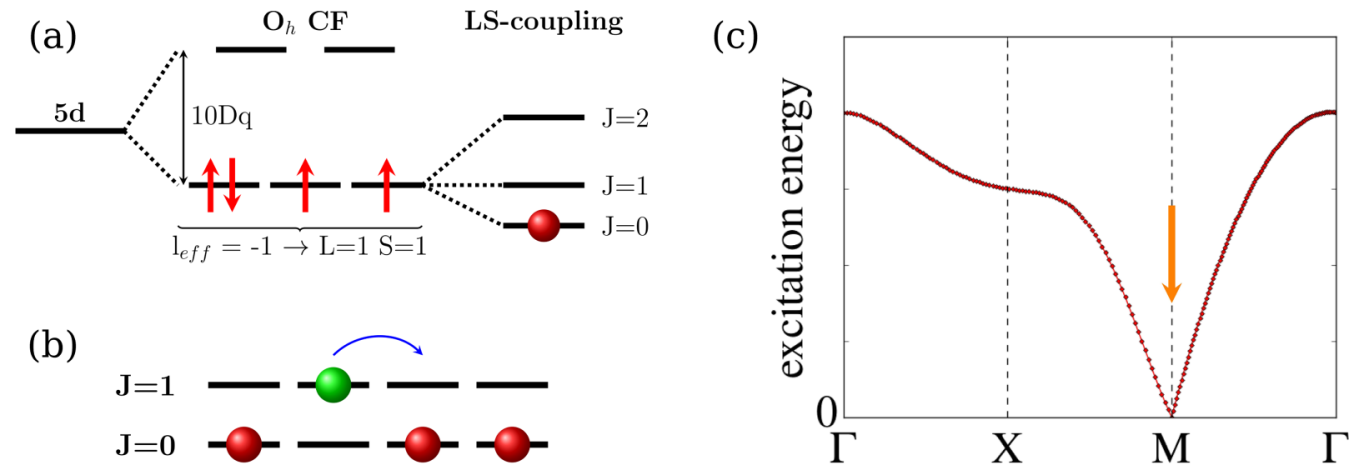

(b)

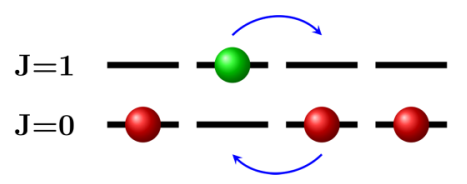

FIG. 1. (a) Schematic illustration of the Ir $d^{4}$ local electronic structure. In an octahedral environment of $O_{h}$ symmetry, the $5 d$ levels split into twofold-degenerate $e_{g}$ and threefold-degenerate $t_{2 g}$ sublevels, which are separated by $10 \mathrm{Dq} \simeq 4 \mathrm{eV}$. This yields an electronic configuration with $L=1$ and $S=1$. The strong SOC gives rise to $J=0$ ground and $J=1, J=2$ excited states, with excitation energies $\gtrsim 0.3 \mathrm{eV}$. (b) Through singlet-triplet site exchange, the $J=1$ exciton can propagate in the background formed by $J=0$ singlets. (c) Schematic illustration of the resulting triplet-mode dispersion on a square lattice (cf. Figs. 3 and 4 in [12]); large "exchange" parameters lead to softening and even condensation of triplet excitations at the $M$ point (equivalent to the $X$ point in the fcc lattice), which induces a transition from a nonmagnetic to a magnetically ordered state.

density-functional-based calculations $[18,19]$ indicate that this is not the driving mechanism. While Bhowal et al. [18] even find a breakdown of the local $J=0$ state due to band-structure effects, Pajskr et al. [19] conclude that the $J=0$ ground state is stable, well "separated" from the $J=1$ level in these double perovskites, preventing any form of intrinsic bulk magnetism.

In order to clarify the issue of excitonic magnetism in $\mathrm{Ba}_{2} \mathrm{YIrO}_{6}$ and $\mathrm{Sr}_{2} \mathrm{YIrO}_{6}$, it is essential to study the momentum dependence of the $J=1$ and $J=2$ excitations in these materials and determine if their dispersions are indeed sufficiently large to result in a condensation of the type sketched in Fig. 1. We therefore investigated the momentum-dependent magnetic excitations, i.e., the spin-orbit excitons, in both materials by means of high-resolution resonant inelastic $x$-ray scattering (RIXS) at the Ir $L_{3}$ edge. We identify strong spin-orbit excitons with $J=1$ and $J=2$ that display a very similar behavior in both materials. Most importantly, the $J=0$ ground state is found to be very stable in $\mathrm{Ba}_{2} \mathrm{YIrO}_{6}$ and $\mathrm{Sr}_{2} \mathrm{YIrO}_{6}$, separated by about $370 \mathrm{meV}$ from the low-lying $J=1$ level. By analyzing the $J=1$ and $J=2$ modes in terms of ab initio quantum chemistry calculations and subsequent effective-Hamiltonian modeling, we extract the relevant energy scales, which indeed exclude the presence of excitonic magnetism in bulk $\mathrm{Sr}_{2} \mathrm{YIrO}_{6}$ and $\mathrm{Ba}_{2} \mathrm{YIrO}_{6}$.

\section{EXPERIMENT}

The RIXS measurements at the Ir $L_{3}$ edge were performed at the beam line ID20 of the European Synchrotron Radiation Facility (ESRF) using $\pi$-polarized photons and a scattering geometry with $2 \theta \simeq 90^{\circ}$ in order to suppress elastic scattering. A spherical, diced $\mathrm{Si}(844)$ analyzer was used in a Rowland circle of $2 \mathrm{~m}$ radius in combination with a custom-built hybrid pixel detector, realizing an overall energy resolution of $\approx 25 \mathrm{meV}$ at the Ir $L_{3}$ edge in this configuration. The incident energy maximizing the resonant enhancement of the features of interest (below $1.5 \mathrm{eV}$ energy loss) was determined to be $11.217 \mathrm{keV}$. The spot size on the sample was approximately $10 \times 20 \mu \mathrm{m}$ and hence significantly smaller than the sample $(\approx 100 \mu \mathrm{m})$. Measurements of the momentum transfer dependence of the $J=1$ and $J=2$ excitation energies were performed at various $q$ points within the fcc Brillouin zone. We note in this context that while $\mathrm{Ba}_{2} \mathrm{YIrO}_{6}$ is perfectly cubic [Fm $\overline{3} m$, cf. Fig. 2(a)], the structure of $\mathrm{Sr}_{2} \mathrm{YIrO}_{6}$ gave rise to some debate. In particular, the previously proposed monoclinic structure $\left(P 2_{1} / n\right)$ [16], which features significant distortions of the $\mathrm{IrO}_{6}$ octahedra, was questioned by a recent study [20] that reports a cubic fcc lattice as in $\mathrm{Ba}_{2} \mathrm{YIrO}_{6}$. The $h k l$ values in the following therefore always refer to the fcc cubic structure of $\mathrm{Ba}_{2} \mathrm{YIrO}_{6}$. The $q$ points were chosen to form paths along high-symmetry directions within the Brillouin zone centered at the $\left(\begin{array}{lll}10 & 0 & 0\end{array}\right)$ Bragg reflection, as depicted in Fig. 2(c). All measurements were performed at room temperature.

\section{RESULTS}

Representative RIXS spectra taken at the $\Gamma$ point of the fcc Brillouin zone are shown in Fig. 2(b) for both $\mathrm{Ba}_{2} \mathrm{YIrO}_{6}$ and $\mathrm{Sr}_{2} \mathrm{YIrO}_{6}$. As seen in the figure, the spectra as a function of the energy loss $\hbar \Omega=E_{i}-E_{f}$ are essentially identical, exhibiting two strong peaks at $\hbar \Omega_{1} \approx 370 \mathrm{meV}$ and $\hbar \Omega_{2} \approx 650 \mathrm{meV}$. Both peaks are resolution limited with a full width at half maximum (FWHM) of less than $30 \mathrm{meV}$. At higher energy losses another weaker feature with $\hbar \Omega_{3} \simeq 1200 \mathrm{meV}$ can be noticed, which appears to be located on top of a broader continuum.

To identify the ground state of the Ir ion and the type of the low-energy intra-5 $d$-shell excitations, we performed $a b$ initio quantum chemistry calculations [21] on an atomic cluster containing one $\mathrm{IrO}_{6}$ octahedron, the six nearest-neighbor $\mathrm{YO}_{6}$ octahedra, and eight adjacent $\mathrm{Ba}^{2+}$ sites, embedded within an effective field that captures the electrostatic effects of the $\mathrm{Ba}_{2} \mathrm{YIrO}_{6}$ ionic lattice. Complete-active-space self-consistentfield (CASSCF) computations with four electrons in three orbitals (the higher-energy valence orbitals of predominant Ir $5 d$ character) were first employed to generate reference wave functions for the subsequent configuration-interaction treatment [21]. The latter were carried out as multireference 
(a)

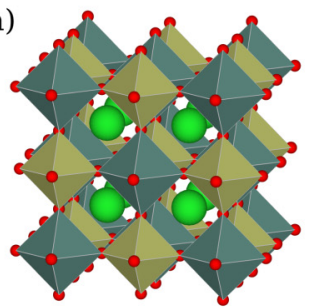

(c)

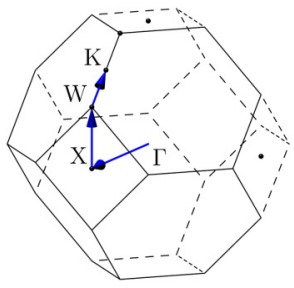

(b)

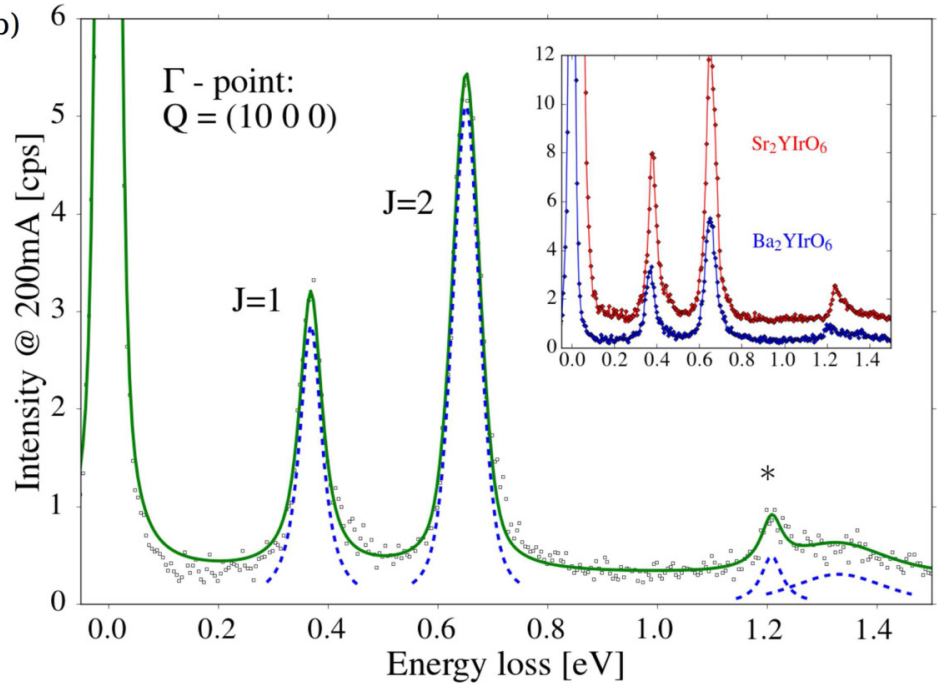

FIG. 2. (a) Unit cell of the cubic double perovskite structure $\left(F m \overline{3} m, a=8.339 \AA\right.$ ). $\mathrm{IrO}_{6}$ and $\mathrm{YO}_{6}$ octahedra are shown in olive and blue, respectively. (b) RIXS spectrum of $\mathrm{Ba}_{2} \mathrm{YIrO}_{6}$ at $Q=(1000)$, obtained with $E_{i}=11.217 \mathrm{keV}$ and room temperature. Two strong features are observed at $370 \mathrm{meV}(J=1)$ and $650 \mathrm{meV}(J=2)$ and a weaker one on top of a continuum around $1200 \mathrm{meV}(*)$. Dashed lines are fits to the observed excitations. Inset: RIXS spectra at $\Gamma$ for $\mathrm{Sr}_{2} \mathrm{YIrO}_{6}$ and $\mathrm{Ba}_{2} \mathrm{YIrO}_{6}$ (the $\mathrm{Sr}_{2} \mathrm{YIrO}_{6}$ spectrum has been background corrected and shifted vertically). (c) The Brillouin zone of the fcc lattice. Blue arrows illustrate the path along which RIXS spectra were collected.

configuration-interaction (MRCI) calculations with single and double excitations out of the $\mathrm{Ir} 5 d$ and $\mathrm{O} 2 p$ shells of the central $\mathrm{IrO}_{6}$ octahedron. SOC was finally included at the quasidegenerate perturbative level in the basis of MRCI states [22]. All calculations were performed using the MOLPRO quantum chemistry package [23]. This type of calculation has previously provided excellent insights into the interplay of $\mathrm{CF}$ splitting, SOC, and ligand-metal hybridization effects for a variety of $4 d$ and $5 d$ compounds [24-27].

Relative energies of the lowest $5 d^{4}$ states are provided in Table I, as obtained by (i) MRCI+SOC computations, (ii) an effective single-ion model (see discussion below), and (iii) from experiment. Only the $t_{2 g}^{4}$ states were explicitly considered in the quantum chemistry calculations, i.e., for the CASSCF optimization and in the spin-orbit MRCI. Our computations indicate a stable spin-orbit singlet ground state, with excitation energies of $0.36-0.37$ and $0.66-0.70 \mathrm{eV}$ to the lowest $J=1$ and $J=2$ components (second and third columns in Table I). These numerical values reproduce very well the positions of the peaks at 0.37 and $0.65 \mathrm{eV}$ in the RIXS experiment [see Fig. 2(b) and the fourth column in Table I]. A second set

TABLE I. Low-energy $\mathrm{Ir}^{5+} 5 d^{4}$ multiplet structure in $\mathrm{Ba}_{2} \mathrm{YIrO}_{6}$, as obtained by ab initio MRCI+SOC calculations, an effective singleion model, and experiment. The RIXS values are the fitted peak positions in Fig. 2(b).

\begin{tabular}{lccc}
\hline \hline$t_{2 g}^{4}$ terms & MRCI + SOC & Model & RIXS \\
\hline${ }^{3} T_{1 g}$ & 0.00 & $0.00(J=0)$ & 0.00 \\
& 0.37 & $0.36(J=1)$ & $0.371 \pm 0.003$ \\
& 0.70 & $0.66(J=2)$ & $0.651 \pm 0.003$ \\
${ }^{1} T_{2 g},{ }^{1} E_{g}$ & $1.71,1.79$ & $1.50(J=2)$ & $\simeq 1.2$ \\
${ }^{1} A_{1 g}$ & 3.09 & $2.72(J=0)$ & \\
\hline \hline
\end{tabular}

of $5 d^{4}$ excited states is found in the range $1.7-1.8 \mathrm{eV}$ in the MRCI+SOC calculations and is mainly related to the ${ }^{1} T_{2 g}$ and ${ }^{1} E_{g} t_{2 g}^{4}$ terms according to our analysis. The agreement between theory and experiment is less appealing in this energy range, since a low-intensity RIXS peak is observed around $1.2 \mathrm{eV}$ in the measurements. On the quantum chemistry side, a better description of the states in this energy window is expected to be achieved by accounting for $t_{2 g}^{4}-t_{2 g}^{4-n} e_{g}^{n}$ and charge-transfer configurational mixing. Since the present study is concerned with states below $1 \mathrm{eV}$ energy loss, these deviations play no role in the following discussion of the sharp and coherent excitations at $\hbar \Omega_{1,2}$.

As already mentioned above, large intersite hoppings may give rise to a strong downward dispersion of the excitonic bands. In other words, as a function of momentum transfer $q$, the relative energy of an excited, magnetic state can be reduced so strongly that it crosses the $J=0$ energy level. We therefore determined experimentally the dispersions of the $J=1$ and $J=2$ modes by means of RIXS. In Fig. 3, RIXS spectra at various $q$ points along the path shown in Fig. 2(c) are presented for $\mathrm{Ba}_{2} \mathrm{YIrO}_{6}$. These data clearly show that the $J=1,2$ excitations, i.e., spin-orbit excitons, exhibit only weak dispersions of less than $50 \mathrm{meV}$, implying high effective masses. The most notable change is a splitting of the $J=2$ signal into two peaks upon moving from $\Gamma$ to the $X, W$, and $K$ points. Most importantly, the observed exciton dispersions show that singlet-triplet site exchange is much too weak in $\mathrm{Ba}_{2} \mathrm{YIrO}_{6}$ to overcome the magnetic excitation gap of about $370 \mathrm{meV}$; i.e., none of the magnetic excitations disperses down to $0 \mathrm{meV}$. Within the error of our experiment the same dispersions are also observed for $\mathrm{Sr}_{2} \mathrm{YIrO}_{6}$ (not shown).

In order to determine the relevant energy scales describing the magnetic system, we introduce an effective Hamiltonian for the spin-orbit exciton propagation, starting from the on-site multiplet structure confirmed at the $a b$ initio 

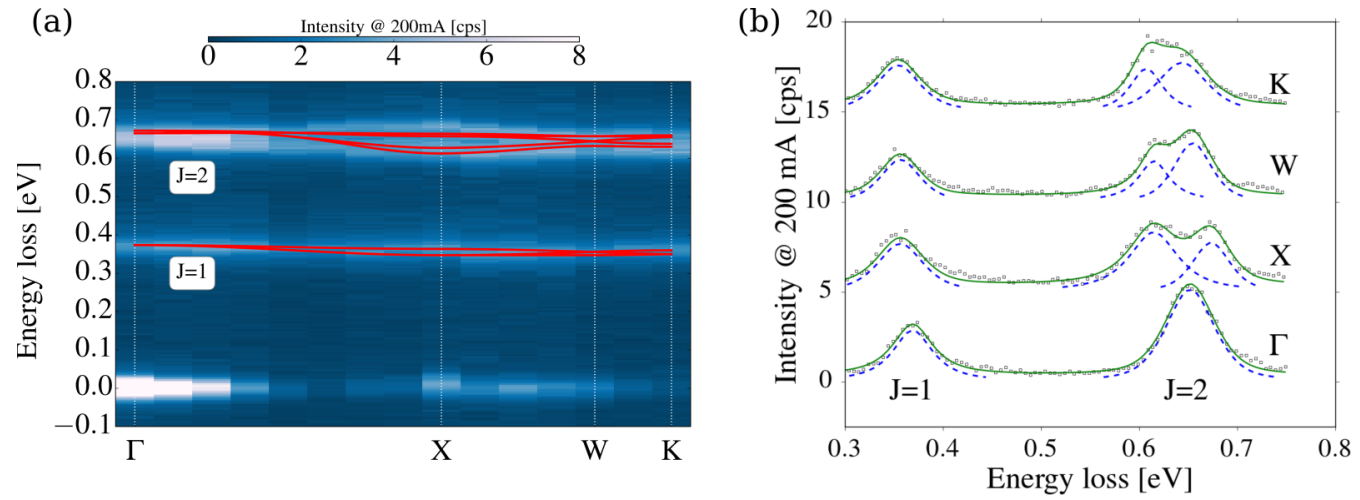

FIG. 3. Momentum-dependent RIXS spectra of $\mathrm{Ba}_{2} \mathrm{YIrO}_{6}$ : (a) False-color plot of the $q$-dependent $J=1$ and $J=2$ excitations extracted from RIXS together with the calculated dispersions for $t=-0.1, U=2, J_{H}=0.4$, and $\lambda=0.43 \mathrm{eV}$ (red lines). (b) Room temperature RIXS spectra taken at the high symmetry points of the fcc Brillouin zone (spectra were shifted by a constant value in intensity for the sake of clarity). For the $J=2$ excitations a splitting is observed at $K, X$, and $W$.

level and by further accounting for $d^{3}-d^{5}$ and $d^{5}-d^{3}$ intersite excitations. The latter can be described in a tightbinding picture through terms of the type $\sum_{\alpha \beta\langle i, j\rangle} t_{\alpha \beta} c_{i \alpha}^{\dagger} c_{j \beta}+$ H.c., where the nearest-neighbor hopping matrix elements between different pairs of Ir $t_{2 g}$ orbitals enter as $t_{\alpha \beta}$. By integrating out the intermediate states, one arrives at an effective "excitonic" Hamiltonian for $J=1$ triplets $(t)$, of the form $\sum_{\alpha \beta \mathbf{k}}\left[\epsilon_{\alpha \beta}(\mathbf{k}) t_{\alpha \mathbf{k}}^{\dagger} t_{\beta \mathbf{k}}+\frac{1}{2}\left(d_{\alpha \beta}(\mathbf{k}) t_{\alpha \mathbf{k}}^{\dagger} t_{\beta-\mathbf{k}}^{\dagger}+\right.\right.$ H.c.) $)$, and for $J=2$ quintet modes $(q)$, with a similar expression $\sum_{\mu \nu \mathbf{k}}\left[\bar{\epsilon}_{\mu \nu}(\mathbf{k}) q_{\mu \mathbf{k}}^{\dagger} q_{\nu \mathbf{k}}+\frac{1}{2}\left(\bar{d}_{\mu \nu}(\mathbf{k}) q_{\mu \mathbf{k}}^{\dagger} q_{\nu-\mathbf{k}}^{\dagger}+\right.\right.$ H.c. $\left.)\right]$, where $\epsilon_{\alpha \beta}$, $\bar{\epsilon}_{\mu \nu}, d_{\alpha \beta}$, and $\bar{d}_{\mu \nu}$ depend on the on-site Coulomb repulsion $U$, the Hund exchange $J_{H}$, the SOC constant $\lambda$, and the hopping matrix element $t$ between nearest-neighbor $\operatorname{Ir} t_{2 g}$ functions with $\sigma$-type mutual orientation. Using a mean-field approximation, we find after a Bogoliubov transformation (see Appendix B) dispersions for both excitonic states. By fits to the experimental data, we obtain the on-site excitation energies listed in the third column of Table I, the curves displayed in red in Fig. 3, and the following values for the relevant effective parameters: $t=-0.1, U=2, J_{H}=0.4$, and $\lambda=0.43 \mathrm{eV}$.

Despite the large spatial extent of the Ir $5 d$ wave functions, we derive a rather small value for the Ir-Ir hopping $t$, which is consistent with the large separation of nearest-neighbor Ir sites in the double-perovskite structure (see Fig. 2). A small $t$ implies stronger correlation effects but also heavier $J=$ 1 "quasiparticles," preventing excitonic magnetism in these double perovskites.

\section{CONCLUSION}

To summarize, we have observed the coherent propagation of heavy $J=1$ and $J=2$ spin-orbit excitons within the background of nonmagnetic $J=0$ sites in $\mathrm{Ba}_{2} \mathrm{YIrO}_{6}$ and $\mathrm{Sr}_{2} \mathrm{YIrO}_{6}$. For both compounds we identify strong and sharp transitions from the $J=0$ ground state to magnetic $J=1$ and $J=2$ excited states at 370 and $650 \mathrm{meV}$, respectively. The $J=1$ and $J=2$ spin-orbit excitons, however, exhibit only a weak energy dispersion of about $50 \mathrm{meV}$. This is far too small to overcome the magnetic excitation gap of $370 \mathrm{meV}$, i.e., the magnetic excitations remain fully gapped, which agrees with recent theoretical results reported by Pajskr et al. [19].

We note that the present measurements have been performed at room temperature, while the experimental evidence for magnetism in these systems has been found at low temperatures. However, our analysis of the relevant effective Hamiltonian shows that the onset of exciton condensation would require an increase of the hopping parameter by a factor of 4 . While this excludes the onset of excitonic magnetism upon cooling in the present materials, it also suggests that this instability can in principle occur in $5 d^{4}$ iridates with $t \simeq 0.4 \mathrm{eV}$. This still requires additional analysis since for $t \simeq \lambda$, however, the ground state may already deviate significantly from the strong spin-orbit-coupling limit used in the present study.

The absence of excitonic magnetism in $\mathrm{Sr}_{2} \mathrm{YIrO}_{6}$ and $\mathrm{Ba}_{2} \mathrm{YIrO}_{6}$ immediately raises the question as to what the origin of the experimentally observed magnetic response in these materials really is. Possible explanations include extrinsic effects due to a small amount of sample imperfections such as oxygen vacancies, resulting in magnetic $\mathrm{Ir}^{4+}$ sites, or intermixing of Ir and Y. This still needs to be clarified in the future. In any case, the present study unambiguously shows that both $\mathrm{Ba}_{2} \mathrm{YIrO}_{6}$ and $\mathrm{Sr}_{2} \mathrm{YIrO}_{6}$ are far from any magnetic instability caused by exciton condensation. This settles the ongoing debate regarding excitonic magnetism in these compounds, implying that neither band-structure effects nor singlet-triplet site exchange are strong enough to cause a breakdown of the $J=0$ ground state in these materials.

\section{ACKNOWLEDGMENTS}

This work has been supported by the Deutsche Forschungsgemeinschaft via SFB 1143. V. M. K., N. A. B., D. E., and L. H. thank Ulrike Nitzsche for technical support.

\section{APPENDIX A: AB INITIO CALCULATION OF THE ON-SITE MULTIPLET STRUCTURE: COMPUTATIONAL DETAILS}

$A b$ initio quantum chemistry calculations were performed on a cluster consisting of one $\mathrm{IrO}_{6}$ reference octahedron 
plus the six $\mathrm{YO}_{6}$ adjacent octahedra and eight nearby $\mathrm{Ba}$ ions. The surrounding solid-state matrix was modeled as a finite array of point charges fitted to reproduce the crystal Madelung field in the cluster region. We used energy-consistent relativistic pseudopotentials for Ir and Y, along with basis sets of quadruple- $\zeta$ [28] and triple- $\zeta$ [29] quality, respectively. Additionally, two $f$ polarization functions were employed for the Ir ion [28]. The oxygen ligands defining the central octahedron were represented by all-electron triple- $\zeta$ basis sets [30], while for the remaining $\mathrm{O}$ sites in our finite cluster $(\mathrm{O}$ ions coordinating the $\mathrm{Y}$ species but not shared with the central $\mathrm{IrO}_{6}$ octahedron) we applied minimal atomic-natural-orbital (ANO) basis functions [31]. For the $\mathrm{Ba}^{2+}$ nearest neighbors we used total-ion effective potentials and a single $s$ valence basis function [32]. One relevant finding in the quantum chemistry calculations is sizable mixing via SOC between the $S=1$ and $S=0 t_{2 g}^{4}$ terms. For this reason, the splitting between the lowest $J=1$ and $J=2$ states is reduced to even slightly less than the $J=0$ to $J=1$ excitation energy, in agreement with experiment (see Table I).

\section{APPENDIX B: THEORY OF EXCITON PROPAGATION}

In order to determine the relevant energy scales describing the magnetic system, we derived explicit expressions for the dispersion of the $J=1$ and $J=2$ modes and analyzed those vis-à-vis experiment. In particular, we considered $d^{3}-d^{5}$ and $d^{5}-d^{3}$ virtual states connected to the $d^{4}-d^{4}$ ground-state configuration through intersite Ir $t_{2 g}-t_{2 g}$ hopping. The tight-bindinglike part of the effective Hamiltonian can be expressed as $H_{t}=$ $\sum_{\alpha \beta\langle i, j\rangle}\left(t_{\alpha \beta} c_{i \alpha}^{\dagger} c_{j \beta}+\right.$ H.c. $)$, where the relevant hopping matrix elements are $t_{\alpha \beta}$. According to density-functional calculations [15], the diagonal effective hopping matrix elements are larger by one order of magnitude than the off-diagonal components; the latter are therefore neglected here and, given the cubic structure, we set $t_{x y, x y}=t_{y z, y z}=t_{z x, z x}=t$. For the simple case of only two nearest-neighbor Ir sites, the spin-orbit part of the effective Hamiltonian can be conveniently written as

$$
H_{\mathrm{SO}}^{i, j}=\sum_{n=0}^{80} E_{n} \frac{\mathcal{P}_{\mathrm{SO}}\left|\Psi_{n}\right\rangle\left\langle\Psi_{n}\right| \mathcal{P}_{\mathrm{SO}}}{\left\langle\Psi_{n}\left|\mathcal{P}_{\mathrm{SO}}^{2}\right| \Psi_{n}\right\rangle},
$$

where $E_{n}$ and $\left|\Psi_{n}\right\rangle$ are eigenvalues and eigenstates in the twosite problem and $\mathcal{P}_{\text {SO }}$ is the projection operator onto the ${ }^{3} T_{2 g} \times$ ${ }^{3} T_{2 g}$ manifold [13,33]. For the extended fcc lattice, this changes to

$$
\begin{aligned}
H_{\mathrm{SO}}= & \sum_{i} \epsilon_{s}\left|s_{i}\right\rangle\left\langle s_{i}\left|+\sum_{i \alpha} \epsilon_{t}\right| t_{\alpha i}\right\rangle\left\langle t_{\alpha i}\left|+\sum_{i \mu} \epsilon_{q}\right| q_{\mu i}\right\rangle\left\langle q_{\mu i}\right| \\
& +\sum_{\langle i, j\rangle \alpha \beta}\left[h_{\alpha \beta}^{i j}\left|s_{i} t_{\alpha j}\right\rangle\left\langle t_{\beta i} s_{j}\left|+d_{\alpha \beta}^{i j}\right| t_{\beta i} t_{\alpha j}\right\rangle\left\langle s_{i} s_{j}\right|+\text { H.c. }\right] \\
& +\sum_{\langle i, j\rangle \mu \nu}\left[h_{\mu \nu}^{i j}\left|s_{i} q_{\mu j}\right\rangle\left\langle q_{\nu i} s_{j}\left|+d_{\mu \nu}^{i j}\right| q_{\nu i} q_{\mu j}\right\rangle\left\langle s_{i} s_{j}\right|+\text { H.c. }\right] \\
& +\cdots,
\end{aligned}
$$

where $\left|s_{i}\right\rangle,\left|t_{\alpha, i}\right\rangle$, and $\left|q_{\mu, i}\right\rangle$ refer to singlet $(J=0)$, triplet $(J=1)$, and quintet $(J=2)$ states at a site $i$, with on-site energies $\epsilon_{s}, \epsilon_{t}$, and $\epsilon_{q}$, respectively, and interaction matri- ces $h_{\alpha \beta}^{i j}=\left\langle s_{i} t_{\alpha j}\left|H_{\mathrm{SO}}^{i j}\right| t_{\beta i} s_{j}\right\rangle, h_{\mu \nu}^{i j}=\left\langle s_{i} q_{\mu j}\left|H_{\mathrm{SO}}^{i j}\right| t_{v i} s_{j}\right\rangle, d_{\alpha \beta}^{i j}=$ $\left\langle t_{\beta i} t_{\alpha j}\left|H_{\mathrm{SO}}^{i j}\right| s_{i} s_{j}\right\rangle$, and $d_{\mu \nu}^{i j}=\left\langle q_{\nu i} q_{\mu j}\left|H_{\mathrm{SO}}^{i j}\right| s_{i} s_{j}\right\rangle$.

Provided that the on-site ground state is a "pure" $J=0$ singlet, the low-lying spin-orbital excitations are characterized by consecutive annihilation of one singlet and creation of one triplet or quintet. One arrives then at an effective excitonic Hamiltonian

$$
\begin{aligned}
H_{\mathrm{ex}}= & \sum_{i \alpha}\left(\epsilon_{t}-\epsilon_{s}\right) T_{\alpha i}^{\dagger} T_{\alpha i}+\sum_{i \mu}\left(\epsilon_{q}-\epsilon_{s}\right) Q_{\mu i}^{\dagger} Q_{\mu i} \\
& +\sum_{\langle i, j\rangle \alpha \beta}\left[h_{\alpha \beta}^{i j} T_{\alpha j}^{\dagger} T_{\beta i}+d_{\alpha \beta}^{i j} T_{\beta i}^{\dagger} T_{\alpha j}^{\dagger}+\text { H.c. }\right] \\
& +\sum_{\langle i, j\rangle \mu \nu}\left[h_{\mu \nu}^{i j} Q_{\mu j}^{\dagger} Q_{\nu i}+d_{\mu \nu}^{i j} Q_{\nu i}^{\dagger} Q_{\mu j}^{\dagger}+\text { H.c. }\right] \\
& +\cdots,
\end{aligned}
$$

where $T_{\alpha i}^{\dagger}=\left|t_{\alpha i}\right\rangle\left\langle s_{i}\right|$ and $Q_{\mu i}^{\dagger}=\left|q_{\mu i}\right\rangle\left\langle s_{i}\right|$. In general, the matrix elements $h_{\alpha \nu}^{i j}=\left\langle s_{i} t_{\alpha j}\left|H_{\mathrm{SO}}^{i j}\right| q_{v i} s_{j}\right\rangle$ are nonzero. They can give rise to coupling between the triplet and quintet excitons $\left(T_{\alpha j}^{\dagger} Q_{\mu i}\right)$. However, the splitting between singlet and quintet $\left(\epsilon_{q}-\epsilon_{t} \approx 0.3 \mathrm{eV}\right)$ is much larger than the coupling constant $h_{\alpha \nu}^{i j}$, since the latter is approximately determined by $\frac{t^{2}}{U}$ and $t$ is very small in $\mathrm{Ba}_{2} \mathrm{YIrO}_{6}$. We therefore neglected this coupling term in our analysis.

Following [3,12], we introduce $\mathbf{T} \rightarrow s^{\dagger} \mathbf{t}$ and $\mathbf{Q} \rightarrow s^{\dagger} \mathbf{q}$, with $n_{s}+n_{t}+n_{q}=1$ for each site. This constraint condition can be taken into account in the mean-field approximation by introducing a Lagrange multiplier. For the ground state $J=0$, the boson operator $s_{i}$ can be treated as a scalar value $s$ with $s^{2}=1$. After transformations as described in [3,12], we end up [34] with

$$
\begin{aligned}
H_{M F} \approx & N \epsilon_{s}+\sum_{\alpha \beta \mathbf{k}}\left[\epsilon_{\alpha \beta}(\mathbf{k}) t_{\alpha \mathbf{k}}^{\dagger} t_{\beta \mathbf{k}}+\frac{1}{2}\left(d_{\alpha \beta}(\mathbf{k}) t_{\alpha \mathbf{k}}^{\dagger} t_{\beta-\mathbf{k}}^{\dagger}+\text { H.c. }\right)\right] \\
& +\sum_{\mu \nu \mathbf{k}}\left[\bar{\epsilon}_{\mu \nu}(\mathbf{k}) q_{\mu \mathbf{k}}^{\dagger} q_{\nu \mathbf{k}}+\frac{1}{2}\left(\bar{d}_{\mu \nu}(\mathbf{k}) q_{\mu \mathbf{k}}^{\dagger} q_{\nu-\mathbf{k}}^{\dagger}+\text { H.c. }\right)\right] \\
& +\cdots,
\end{aligned}
$$

where

$$
\begin{aligned}
& \epsilon_{\alpha \beta}(\mathbf{k})=\left(\epsilon_{t}-\epsilon_{s}\right) \delta_{\alpha \beta}+\sum_{\delta} h_{\alpha \beta}^{\delta} e^{i \mathbf{k} \cdot \mathbf{r}_{\delta}}, \\
& d_{\alpha \beta}(\mathbf{k})=\sum_{\delta} d_{\alpha \beta}^{\delta} e^{i \mathbf{k} \cdot \mathbf{r}_{\delta}}, \\
& \bar{\epsilon}_{\mu \nu}(\mathbf{k})=\left(\epsilon_{q}-\epsilon_{s}\right) \delta_{\mu \nu}+\sum_{\delta} \bar{h}_{\mu \nu}^{\delta} e^{i \mathbf{k} \cdot \mathbf{r}_{\delta}}, \\
& \bar{d}_{\mu \nu}(\mathbf{k})=\sum_{\delta} \bar{d}_{\mu \nu}^{\delta} e^{i \mathbf{k} \cdot \mathbf{r}_{\delta}} .
\end{aligned}
$$

With a subsequent Bogoliubov transformation, one can easily obtain the dependencies provided in Fig. 3. 
[1] G. Jackeli and G. Khaliullin, Phys. Rev. Lett. 102, 017205 (2009).

[2] J. Chaloupka, G. Jackeli, and G. Khaliullin, Phys. Rev. Lett. 105, 027204 (2010).

[3] G. Khaliullin, Phys. Rev. Lett. 111, 197201 (2013).

[4] A. Kitaev, Ann. Phys. 321, 2 (2006).

[5] B. Kim, H. Ohsumi, T. Komesu, S. Sakai, T. Morita, H. Takagi, and T.-h. Arima, Science 323, 1329 (2009).

[6] D. Pesin and L. Balents, Nat. Phys. 6, 376 (2010).

[7] J. Maciejko, V. Chua, and G. A. Fiete, Phys. Rev. Lett. 112, 016404 (2014).

[8] F. Wang and T. Senthil, Phys. Rev. Lett. 106, 136402 (2011).

[9] Y. K. Kim, N. H. Sung, J. D. Denlinger, and B. J. Kim, Nat. Phys. 12, 37 (2016).

[10] X. Wan, Phys. Rev. B 83, 205201 (2011).

[11] W. Witczak-Krempa, A. Go, and Y. B. Kim, Phys. Rev. B 87, 155101 (2013).

[12] A. Akbari and G. Khaliullin, Phys. Rev. B 90, 035137 (2014).

[13] B. H. Kim, G. Khaliullin, and B. I. Min, Phys. Rev. Lett. 109, 167205 (2012).

[14] Y. Kuramoto, H. Kusunose, and A. Kiss, J. Phys. Soc. Jpn. 78, 072001 (2009).

[15] T. Dey, A. Maljuk, D. V. Efremov, O. Kataeva, S. Gass, C. G. F. Blum, F. Steckel, D. Gruner, T. Ritschel, A. U. B. Wolter, J. Geck, C. Hess, K. Koepernik, J. van den Brink, S. Wurmehl, and B. Büchner, Phys. Rev. B 93, 014434 (2016).

[16] G. Cao, T. F. Qi, L. Li, J. Terzic, S. J. Yuan, L. E. DeLong, G. Murthy, and R. K. Kaul, Phys. Rev. Lett. 112, 056402 (2014).

[17] J. Terzic, H. Zheng, F. Ye, H. D. Zhao, P. Schlottmann, L. E. De Long, S. J. Yuan, and G. Cao, Phys. Rev. B 96, 064436 (2017).

[18] S. Bhowal, S. Baidya, I. Dasgupta, and T. Saha-Dasgupta, Phys. Rev. B 92, 121113 (2015).

[19] K. Pajskr, P. Novák, V. Pokorný, J. Kolorenč, R. Arita, and J. Kuneš, Phys. Rev. B 93, 035129 (2016).
[20] L. T. Corredor, G. Aslan-Cansever, M. Sturza, K. Manna, A. Maljuk, S. Gass, T. Dey, A. U. B. Wolter, O. Kataeva, A. Zimmermann, M. Geyer, C. G. F. Blum, S. Wurmehl, and B. Büchner, Phys. Rev. B 95, 064418 (2017).

[21] T. Helgaker, P. Jörgensen, and J. Olsen, Molecular ElectronicStructure Theory (Wiley, Chichester, U.K., 2000).

[22] A. Berning, M. Schweizer, H.-J. Werner, P. J. Knowles, and P. Palmieri, Mol. Phys. 98, 1823 (2000).

[23] H.-J. Werner, P. J. Knowles, G. Knizia, F. R. Manby, and M. Schütz, Wiley Interdiscip. Rev.: Comput. Mol. Sci. 2, 242 (2012).

[24] V. M. Katukuri, K. Roszeitis, V. Yushankhai, A. Mitrushchenkov, H. Stoll, M. van Veenendaal, P. Fulde, J. van den Brink, and L. Hozoi, Inorg. Chem. 53, 4833 (2014).

[25] N. A. Bogdanov, V. M. Katukuri, J. Romhányi, V. Yushankhai, V. Kataev, B. Büchner, J. van den Brink, and L. Hozoi, Nat. Commun. 6, 7306 (2015).

[26] R. Yadav, N. A. Bogdanov, V. M. Katukuri, S. Nishimoto, J. van den Brink, and L. Hozoi, Sci. Rep. 6, 37925 (2016).

[27] L. Xu, N. A. Bogdanov, A. Princep, P. Fulde, J. van den Brink, and L. Hozoi, Quantum Mater. 1, 16029 (2016).

[28] D. Figgen, K. A. Peterson, M. Dolg, and H. Stoll, J. Chem. Phys. 130, 164108 (2009).

[29] K. A. Peterson, D. Figgen, M. Dolg, and H. Stoll, J. Chem. Phys. 126, 124101 (2007).

[30] T. H. Dunning, J. Chem. Phys. 90, 1007 (1989).

[31] K. Pierloot, B. Dumez, P.-O. Widmark, and B. Roos, Theor. Chim. Acta 90, 87 (1995).

[32] P. Fuentealba, L. von Szentpaly, H. Preuss, and H. Stoll, J. Phys. B 18, 1287 (1985).

[33] S. M. Winter, Y. Li, H. O. Jeschke, and R. Valentí, Phys. Rev. B 93, 214431 (2016).

[34] B. H. Kim, D. V. Efremov, and J. v. d. Brink (unpublished). 